\title{
Study protocol medical rehabilitation after COVID-19 disease: an observational study with a comparison group with obstructive airway disease / Re_Co
}

Mercedes Rutsch ${ }^{*}$, Jördis Frommhold ${ }^{2}$, Heike Buhr-Schinner ${ }^{3}$, Levan Djeiranachvili ${ }^{4}$, Thomas Gross ${ }^{5}$, Per Otto Schüller ${ }^{6}$, Alexander Katalinic ${ }^{1}$ and Ruth Deck ${ }^{1}$

\begin{abstract}
Background: Novel coronavirus disease 2019 (COVID-19) has been the subject of a numerous research projects over the past year. In cases with a severe disease course or threatening long-term impairment due to disease, the German health care system offers insured persons the possibility of medical rehabilitation. In contrast to what was observed and expected at the beginning of the pandemic, COVID-19 patients with varying degrees of disease severity are represented in rehabilitation. To date, there is no common consensus on the content and aftercare of rehabilitation nor is there any knowledge about the short- and long-term effects of such a rehabilitation programme. In addition, these aspects were not considered with regard to the varying severity of the course of the disease. The present research project investigates this question.

Methods: The study sample will consist of $N=350$ rehabilitants after COVID-19 and a comparison group (CG) with $N=230$ rehabilitants suffering from an obstructive respiratory disease. The participants will be recruited at five German rehabilitation facilities and undergo medical rehabilitation. This prospective, exploratory, multicentre, mixed-methods study will be evaluated as follows: (A) The quantitative portion includes questionnaires at different points in time (at the beginning and end of rehabilitation, after six and twelve months) and contains standardised measurement instruments. For example, participation limitations, quality of life, health status, fatigue, psychomental limitations and disorders, performance in different areas of life and ability to work are measured. (B) Qualitative interviews are held at different times (end of rehabilitation, after six and twelve months), and an expert workshop is conducted. Topics are rehabilitation content, satisfaction and aftercare as well as different outcomes on subjective health and participation impairments.

(Continued on next page)
\end{abstract}

\footnotetext{
* Correspondence: mercedes.rutsch@uksh.de

${ }^{1}$ Institute for Social Medicine and Epidemiology, Department Rehabilitation

Sciences, University of Lübeck, Ratzeburger Allee 160, 23562 Lübeck,

Germany

Full list of author information is available at the end of the article
}

(c) The Author(s). 2021 Open Access This article is licensed under a Creative Commons Attribution 4.0 International License, which permits use, sharing, adaptation, distribution and reproduction in any medium or format, as long as you give appropriate credit to the original author(s) and the source, provide a link to the Creative Commons licence, and indicate if changes were made. The images or other third party material in this article are included in the article's Creative Commons licence, unless indicated otherwise in a credit line to the material. If material is not included in the article's Creative Commons licence and your intended use is not permitted by statutory regulation or exceeds the permitted use, you will need to obtain permission directly from the copyright holder. To view a copy of this licence, visit http://creativecommons.org/licenses/by/4.0/ The Creative Commons Public Domain Dedication waiver (http://creativecommons.org/publicdomain/zero/1.0/) applies to the data made available in this article, unless otherwise stated in a credit line to the data. 
(Continued from previous page)

Discussion: Studies on other indications have already shown that pneumological rehabilitation has positive effects. Thus, it is expected that an improvement in different dimensions will be observed at the end of rehabilitation in both groups. With regard to the different severities of COVID-19, this study evaluates the long-term developments. Subsequently, the authors will elaborate concrete recommendations for medical rehabilitation after different courses of disease with regard to existing pneumological rehabilitation concepts.

Trial Registration: German Register of Clinical Trials, www.drks.de.Identifier: DRKS00023642; Registered: 01.12.2020.Date and version identifier: 08.04.2021; version 4.

Keywords: Coronavirus disease, COVID-19, Rehabilitation, Post-acute

\section{Background}

COVID-19 is caused by the SARS-CoV-2 virus and is characterised by an unspecific, varying and diverse course of the disease, ranging from the lack of symptoms to severe pneumonia with lung failure and death [1]. The majority of patients have a mild to moderate course of disease (approximately $81 \%$ ) [2]. As a result, approximately $18 \%$ were hospitalised in the first wave of the disease [3]. However, a moderate course of COVID-19 does not equate to a fast recovery. Patients with a severe course of the disease or those who have long-lasting symptoms require further treatment [4].

There are currently no uniform definitions of the long-term effects of COVID-19 ("long COVID") [1]. Especially in very mild or asymptomatic courses, little is known to date. The Robert Koch Institute (RKI) refers to studies of SARS-CoV-1 infection to obtain information about the long-term consequences of COVID-19. According to this information, long-term physical and psychological consequences could be expected for COVID-19 patients. RKI refers to studies that report persistent impairment of lung function $[5,6]$, reduced physical performance $[5,6]$, reduced health-related quality of life $[5,7]$ and various mental and neuropsychiatric impairments, such as anxiety disorders, panic attacks, depression, post-traumatic stress disorder [7]. Such evidence for COVID-19 survivors is currently only found in a few studies with short- to medium-term follow-up. In an Italian study, 143 hospitalised COVID-19 patients were questioned 60 days after their first symptoms. Only $13 \%$ of the respondents stated that they were completely symptom free. In contrast, $32 \%$ reported one or two symptoms, and $55 \%$ reported three or more symptoms. Half of the respondents (53\%) complained of fatigue, $43 \%$ of dyspnoea, $27 \%$ of joint pain and $22 \%$ of chest pain [8]. In another study conducted by King's College London, the UK Covid-19 Symptom Study App assessed the long-term effects of the disease [9]. One in ten users reported persistent symptoms even after three weeks. Symptoms included fatigue, headache, cough, loss of smell, sore throat, delirium and chest pain.
Due to suspected long-term consequences of COVID19 , the literature provides recommendations for physiotherapeutic treatment and early rehabilitation $[10,11]$ and calls for scientific studies on the topic of rehabilitation with COVID-19 [12]. Social reintegration after coronavirus disease should be of particular importance in the post-acute phase [13] as well as for patients with a mild disease course but long-lasting symptoms. Therefore, a multi-professional and interdisciplinary rehabilitation approach is recommended for moderate and severe disease progression [14, 15]. According to recent publications, there is a consensus that multimodal and interdisciplinary pneumological rehabilitation can be described as an effective therapeutic approach [14]. It is necessary to analyse the pulmonary, physical, psychosocial and cognitive dimensions of the disease within scientific studies [16] and to identify individual rehabilitation strategies based on this analysis [12].

Case reports in social networks and on public television show that the long-term effects of COVID-19 will have to be scientifically investigated in the future. These reports also demonstrate that persistent symptoms can also diverge from a linear course and vary in intensity and impairment over time. As a result, on the one hand, persons with a severe course of the disease can be found in the context of follow-up rehabilitation. On the other hand, patients with mild or moderate disease progression are impaired by long-lasting symptoms and can improve their occupational and social participation disorder through rehabilitation.

Given the novelty of the disease, there is no uniform approach to medical rehabilitation after COVID-19. Neither the rehabilitation content nor outcomes are known. In addition, the aftercare that is offered, recommended and received is also unknown. This observational study will provide recommendations for action, include a comparison group and consider the severity of COVID-19.

\section{Aims}

The aim of our study is to describe the health status after COVID-19 in the context of medical rehabilitation 
and its medium- and long-term effects in different dimensions. For this purpose, the study examines the burden of disease and changes in health status under medical rehabilitation and after six and twelve months. Based on the experience of studies on pneumological rehabilitation, we expect positive effects on the general state of health and psychomental disorders at the end of rehabilitation. At follow-up, we expect that in addition to the outcomes already mentioned, rehabilitation success is represented in the dimensions of participation, quality of life, performance in various areas of life, ability to work, and subjective prognosis of employment.

Including rehabilitants from different clinics, it is possible to describe different therapeutic approaches, groups of patients and courses of disease. Statements are made about the extent to which existing rehabilitation approaches meet the needs of COVID-19 rehabilitants, the extent to which new rehabilitation therapy concepts have to be developed and how these are constituted. To date, it is unclear which requirements rehabilitants have with regard to aftercare, which recommendations rehabilitation clinics make and to what extent rehabilitants implement them.

The analyses also considered the severity of the disease course and compared the study results with a reference group with obstructive airway disease (COPD or bronchial asthma). Finally, the study will provide concrete recommendations for rehabilitation after COVID-19.

\section{Intervention}

As an observational study, there is no intervention in the strict sense. The rehabilitants receive medical rehabilitation directly following hospitalisation or with greater time after SARS-CoV-2 infection. The duration of rehabilitation is 21 days with the possibility of extension. Given that the study participants are recruited from different clinics, the rehabilitation programmes differ in terms of therapy concept and structure.

At the MEDIAN Klinik Heiligendamm, the therapeutic elements of rehabilitation include general strengthening, mobilisation and normalisation of respiratory functions. This is intended to improve respiratory distress, respiratory muscle strength, pulmonary resilience, psychomental impairments and/or stress-induced mental disorders of the disease. The main components of the therapy include respiratory gymnastics with respiratory muscle training as well as dissolving phlegm. If required, group discussions (1-2 h per week) are offered to exchange experiences of coronavirus disease, relaxation training and supportive psychological counselling to cope with trauma, fear or depression.

The Reha-Zenrtum Schömberg treats patients in the pneumology department after severe respiratory insufficiency caused by a respiratory disease (including bronchial asthma and COPD) and after pneumonia. With this experience, the rehabilitation centre also receives COVID-19 patients for rehabilitation. The therapy contents include physical and balneotherapy, physiotherapy and exercise therapy, occupational therapy and psychological support.

Ostseeklinik Schönberg-Holm offers medical rehabilitation for various respiratory diseases. These illnesses range from bronchial asthma and COPD to the novel disease COVID-19. This facility also pursues a multimodal and holistic treatment approach during pneumological rehabilitation.

The comparison group is recruited in the MEDIAN Klinik Flechtingen and MEDIAN Klinik Flachsheide Bad Salzuflen as well as in the Ostseeklinik Schönberg-Holm and Reha-Zentrum Schömberg. The CG consists of COPD and asthma bronchial patients. These patients receive rehabilitation and aftercare according to the standards of the German Pension Insurance. The rehabilitants receive treatments comparable to those after COVID-19. Depending on their needs, the rehabilitation includes physical training (respiratory gymnastics, functional gymnastics, ergometer training, walking training, weight training, and treadmill training), respiratory physiotherapy, respiratory therapy, education, psychological interventions, nutritional therapy, counselling by social services and inhalation.

The particular therapeutic approaches are documented and described within the study.

\section{Methods}

\section{Eligibility criteria}

Rehabilitants after varying degrees of COVID-19 who are able to work will be recruited to the study. The rehabilitants may no longer remain in isolation. Participants are between 18 and 65 years old and provide written consent. Participants in the control group have a diagnosis of obstructive airway disease, such as bronchial asthma or COPD. This group will be similar based on age, sex and ability to work.

Individuals who do not provide consent and who do not have sufficient knowledge of German are excluded. Furthermore, rehabilitation may not be paid by German Social Accident Insurance.

\section{Study design}

This is a prospective, explorative, longitudinal multicentre observational study without any specific intervention. Different methodological components (mixed-method study; see sections A and B) are included. The aim of the study is to obtain an overview of the problems associated with the new disease COVID-19 to derive recommendations for future clinical practice. 
The Institute for Social Medicine and Epidemiology at the University of Lübeck leads the study. Consecutive recruitment will occur in five different rehabilitation clinics in Germany (MEDIAN Klinik Heiligendamm, Reha-Zentrum Schömberg, Ostseeklinik SchönbergHolm, MEDIAN Klinik Flachsheide Bad Salzuflen, MEDIAN Klinik Flechtingen). Each clinic designates a study representative who recruits suitable rehabilitants during their first days in rehabilitation. If possible, the study representatives personally contacted the study participants and provides them with the information material and the declaration of consent.

\section{A: quantitative measures}

To measure the long-term burden of COVID-19 and the effects of rehabilitation, the study includes four measurement points: at the beginning $\left(t_{0}\right)$, at the end of rehabilitation $\left(t_{1}\right)$, six $\left(t_{2}\right)$ and twelve months after rehabilitation $\left(t_{3}\right)$. A questionnaire with standardised and validated instruments will be completed by all study participants at each measurement point.

In the rehabilitation clinics, the participants of both groups are recruited consecutively at the same time until the planned number of cases is reached.

\section{Outcomes}

Based on the International Classification of Functioning, Disability and Health (ICF), the IMET [17] measures the restrictions for social and work-related participation of persons with a chronic disease $[18,19]$. The IMET is a valid instrument and assesses the limitations of participation in nine relevant dimensions of daily life. On a scale from 0 to 10 , the respondent ranks how high their limitations are in each dimension. A high score is associated with limited participation. Both, individual items and the cumulative score can be evaluated.

The VR-12 measures health-related quality of life (HRQoL) at the beginning of rehabilitation and at catamnesis. The VR-12 is based on the SF-36 and the Veterans Health Study. It covers eight health domains, and a total score is provided [20]. We use the translated and culturally adapted German version, which was validated in a German sample of inpatient rehabilitation patients [21].

Core symptoms of depression, anxiety and stress are assessed with the DASS. The instrument is mainly used in patients with pain, but it is also used for patients without pain-specific symptoms. The short version in Germany comprises 21 items and 7 items for the respective scales of depression, anxiety and stress. The individual scales of the DASS have sufficient to good reliability and have also been classified as valid [22].

The presence of a generalised anxiety disorder as well as its symptom severity is measured using the
GAD-7 [23]. For this purpose, 7 items are used according to the most important diagnostic criteria of generalised anxiety disorder as defined in the Diagnostic and Statistical Manual of Mental Disorders (DSM-IV). The response options from "not at all" to "nearly every day" indicate how often the problem occurred in the last two weeks. The scale has good reliability and validity.

Depressiveness is measured with the PHQ-9 [24], which is the depression module of the Patient Health Questionnaire (PHQ-D) and contains nine questions. Each item captures one of the nine DSM-IV criteria of "major depression". According to a large-scale metaanalysis, the PHQ-9 has very good specificity and sensitivity [25].

The COPD Assessment Test (CAT) [26] assesses the effects of chronic obstructive pulmonary disease. The CAT is a reliable instrument designed to measure the impact of COPD on health status and daily life [27]. A semantic six-point differential scale is used for the eight items. Cough, phlegm, tightness, breathlessness going up hills/stairs, activity limitation at home, confidence leaving home, sleep and energy are measured with one item each.

The Work Ability Score (WAS) of the Work Ability Index (WAI) measures the work ability. In numerous studies, this score provided acceptable values in terms of validity and reliability [28]. The higher the score on the scale from 0 to 10 , the higher the ability to work.

The German version of the SPE scale is used to predict the subjective prognosis of employment. To divide the study participants into two risk groups, the respondents assess their occupational risk with three items from which an overall score is formed. The validity and reliability of the SPE scale was positively evaluated in a large sample [29].

Using individual items, occupational changes are measured, including job retention interventions and types of job loss [30].

Fatigue is measured with the EORTC-FA12, which has been validated in large international and multicentre studies [31]. With the help of twelve four-stage items ("not at all" to "very much"), physical, emotional and cognitive impairments caused by fatigue are assessed. In a German study validating the questionnaire with breast cancer patients [32], a high consistency of the overall scale was observed; the subscale also shows good to high internal consistency [32].

Single items without standardisation capture COVID19-associated life events. Questions are asked about the death of a relative due to COVID-19, COVID-19associated physical and/or social deprivation, loss of autonomy and loss of economic livelihood. Participants are also asked if they infected someone else, how they feel 
about it and if they feel that their general practitioner takes their symptoms seriously.

Performance in different areas of life is assessed with three scales from 0 to 10 for the areas of work, everyday life and leisure. The individual scales are valid instruments for determining performance limitations. A low sum score indicates a high level of performance limitations [30, 33].

To assess the extent of physical activity, questions are asked in conjunction with Menski's Federal Health Surveys [34].

The moderating variables include comorbidity [35], risk factors and sociodemographic variables [36].

The rehabilitants receive the questionnaires at four measurement points. Some of the outcomes that are not likely to change during rehabilitation are only measured at the beginning of rehabilitation and at catamnesis. These are parameters that probably change only insignificantly during rehabilitation (e.g., comorbidities) or outcomes addressing everyday life and the domestic environment. Table 1 presents the set of core instruments used in the study.

\section{B: qualitative measures}

Telephone interviews and an expert workshop occur during the study. The guidelines for the telephone interview were pre-tested with COVID-19 rehabilitants.
Rehabilitants provided feedback on the comprehensibility of the questions and the length of the interview.

Regarding telephone interviews, nine rehabilitants after COVID-19 were interviewed at three points in time: after medical rehabilitation, six and twelve months after rehabilitation. In the comparison group, eight interviews were held six months after rehabilitation. The interview participants varied in terms of sex, age and educational background.

In the semi-structured interviews, the participants were asked about the course of their disease, the contents and goals of their rehabilitation, their satisfaction, their current disease burden, their social and occupational participation and their rehabilitation aftercare.

The expert workshop involves clinical staff, funding agencies and scientists. The topics of the workshop include the rehabilitation procedure, general conditions of rehabilitation after COVID-19, and existing pneumological therapy concepts in rehabilitation. Finally, recommendations for rehabilitation after COVID-19 are formulated.

Both the interviews and the expert workshop follow guidelines. The guidelines and the results of the qualitative research are discussed by the project team and at the brain trust "Arbeitsgruppe qualitative Methoden" (AQUAM). The interviews last approximately $30 \mathrm{~min}$, while the expert workshop is correspondingly longer. With the agreement of all participants, the interviews and the workshop are recorded and transcribed word by

Table 1 Core set of instruments used in the quantitative part of the study

\begin{tabular}{|c|c|c|c|c|c|}
\hline Dimensions & Instruments & $t_{0}$ & $t_{1}$ & $t_{2}$ & $t_{3}$ \\
\hline Restriction of Participation & IMET [17] & $\bullet$ & & $\bullet$ & $\bullet$ \\
\hline Quality of Life & VR12 [20] & $\bullet$ & & $\bullet$ & $\bullet$ \\
\hline Depression, Anxiety and Stress Scale & DASS [22] & $\bullet$ & $\bullet$ & $\bullet$ & $\bullet$ \\
\hline Stress-Related Psychomental Limitations and Disorders & GAD-7 [23]; PHQ-9 [24] & $\bullet$ & $\bullet$ & $\bullet$ & $\bullet$ \\
\hline Corona-associated Life Events & Single Items & $\bullet$ & $\bullet$ & $\bullet$ & $\bullet$ \\
\hline Effects of Chronic Obstructive Pulmonary Disease & CAT [26] & $\bullet$ & $\bullet$ & $\bullet$ & $\bullet$ \\
\hline Fatigue & EORTC-FA 12 [32] & $\bullet$ & $\bullet$ & $\bullet$ & $\bullet$ \\
\hline General State of Health & Single Items & $\bullet$ & $\bullet$ & $\bullet$ & $\bullet$ \\
\hline Performance in Various Areas of Life & QGmR [30] & $\bullet$ & & $\bullet$ & $\bullet$ \\
\hline Occupational Changes & $\begin{array}{l}\text { Single Items from } \\
\text { QGmR [30] }\end{array}$ & & & $\bullet$ & $\bullet$ \\
\hline Occupational Situation & Single Items & $\bullet$ & & $\bullet$ & $\bullet$ \\
\hline Ability to Work & $\begin{array}{l}\text { Work Ability Score, } \\
\text { WAS [28] }\end{array}$ & $\bullet$ & & $\bullet$ & $\bullet$ \\
\hline Subjective Prognosis of Employment & SPE-Scale [29] & $\bullet$ & & $\bullet$ & $\bullet$ \\
\hline \multicolumn{6}{|l|}{ Moderating Variables } \\
\hline Comorbidity & Single Items [35] & $\bullet$ & & $\bullet$ & $\bullet$ \\
\hline Risk Factors & Single Items & $\bullet$ & $\bullet$ & $\bullet$ & $\bullet$ \\
\hline Sociodemographic Data & Single Items [36] & $\bullet$ & & $\bullet$ & $\bullet$ \\
\hline
\end{tabular}

$t_{0}$ baseline/right before rehabilitation; $t_{1}$ 3-week follow-up/right after rehabilitation; $t_{2} 6$ months after rehabilitation, $t_{3} 12$ months after rehabilitation 
word. The evaluation of data is based on qualitative content analysis.

\section{Data management Patient documentation}

Study participants are uniquely organised and documented in all clinics using an automated documentation template. All study representatives are briefed on the template before recruitment.

The included study participants receive an identification number (ID) to pseudonymise their personal data. The first number is used to classify whether the participant receives rehabilitation after COVID-19 or belongs to the comparison group. The second number of the ID refers to the rehabilitation clinic where the participant is recruited. The subsequent numbering corresponds to the number of recruited participants. Personal data are only documented in a separate Excel file; appropriate linkage occurs using the ID. The data will not be sent to the ISE until the personal contents are deleted. This collection includes only standardised documentation in the clinics. The data from the questionnaire are collected and kept separately from the ISE.

\section{Data entry and control}

The data for the quantitative part of the study (A) are generated by standardised written questionnaires. The answers to the questionnaires are entered into SPSS 22.0 by a student assistant under the guidance of the research assistant. The entered data are assessed for random duplicate entries $(5-10 \%)$ by checking their validity and plausibility.

\section{Data monitoring}

\section{Data monitoring in the clinics is not planned}

Case number calculation/sample size To determine an appropriate sample size, it is assumed that the indicators used to characterise the sample should be described with sufficient precision. Sufficient precision should be applied to continuous indicators if the range of the $95 \%$ confidence interval around a sample mean does not exceed a pre-specified value. For categorical variables, it is analogously assumed that the range of the $95 \%$ confidence interval should not exceed a pre-specified value by a sample proportion. In the case of a continuous variable, the limits mentioned are set at $+/-5$ points for a scale with a value range of 0 to 100 . In the case of proportions, the range of the $95 \%$ confidence interval should not exceed $+/-10 \%$ points in the range of percentages from 10 to $40 \%$. The required width of the $95 \%$ confidence interval is achieved with a continuous variable from a sample size of $\mathrm{N}=100$. In the case of a binary variable in the range between 10 and $40 \%$, the width of the $95 \%$ confidence interval will be less than $20 \%$ points for sample sizes of 100 or more. To compensate for smaller deviations in the required number of cases, a $20 \%$ larger sample is taken compared to this net number of cases, i.e., $\mathrm{N}=120$ cases. We also expect a dropout rate of $20 \%$ [37, 38]. Thus, the MEDIAN Klinik Heiligendamm will recruit $\mathrm{N}=150$, and the RehaZentrum Schömberg and the Ostseeklinik SchönbergHolm will separately recruit $\mathrm{N}=100$ COVID-19 rehabilitants. For the CG, the MEDIAN Klinik Flechtingen and Bad Salzuflen will recruit $N=75$ rehabilitation patients, separately, and the Reha-Zentrum Schömberg and Ostseeklinik Schönberg-Holm will recruit $\mathrm{N}=40$ rehabilitants per clinic.

\section{Statistical methods Quantitative analysis}

In the course of the study, no predefined hypotheses are confirmed. Rather, the rehabilitation groups are precisely characterised. Comparative analyses are used to determine the relevant differences between the subgroups and the comparison group. Accordingly, we mainly provide descriptive evaluations on the burden of disease as well as on health-related changes during and after rehabilitation.

For the interval-scaled variables, variance analyses with measurement repetition are calculated to evaluate the pre-post differences of the outcomes at the various measurement times. Depending on the data constellation, the t-test for dependent or independent samples is used as a parametric significance test. Non-parametric tests are performed for ordinally scaled data. Subgroup analyses will be planned for sex, age and education as well as risk factors and comorbidities. To classify the findings in COVID-19 patients, the stress manifestations and the measured changes are compared with those in the CG.

In addition to the analysis of the health-related changes in relation to a comparison group, the treatment concepts from different rehabilitation facilities are also examined. The type and extent of the therapy, characteristics of the included rehabilitants, perceived benefits of the rehabilitation and subjectively experienced health improvements are recorded. All calculations are performed with SPSS 22.0.

\section{Qualitative analysis of the interviews and expert workshop}

The interviews and focus group are analysed using qualitative content analysis [39-41]. We use MAXQDA 12 for coding. The transcripts are checked for relevant topics and interesting content to finally develop a category system. The main topics are derived from the research questions and the interview guide; subcategories 
are induced from materials developed, e.g., in subsumption [40]. To test the category system, independent test coding is performed by two researchers; then, the categories and definitions are modified. The entire coding process is developed in the form of a consensual coding [42], i.e., the transcriptions are coded independently by two scientists. Finally, the coding is consolidated to develop a consensus.

\section{Discussion}

The novelty of the disease and the increasing use of rehabilitation services by COVID-19 patients enhances the call for research. At present, COVID-19 rehabilitation does not follow uniform guidelines. Therefore, it is necessary to review the different therapeutic approaches and to formulate recommendations for pneumological rehabilitation after COVID-19. Furthermore, the rehabilitation needs of COVID-19 patients and their rehabilitation capacity will be assessed. The concrete short-, medium- and long-term effects of COVID-19 medical rehabilitation have not yet been evaluated.

When assessing rehabilitation, it is also essential to analyse rehabilitation aftercare. To date, there is no evidence on the needs at the end of rehabilitation, nor is there a uniform approach to rehabilitation aftercare. This study clarifies which aftercare recommendations are given to these rehabilitants, to what extent they are realised and how the needs of the rehabilitants are met.

It now appears that COVID-19 survivors of any disease severity seek rehabilitation. Therefore, the study results are also differentiated against the different degrees of severity and additionally contrasted with a comparison group. Finally, recommendations for rehabilitation and rehabilitation aftercare need to be developed for COVID-19 patients.

\section{Innovation factor}

During the telephone interviews and written survey, the study participants are asked about their aftercare recommendations, the use of aftercare programmes and their wishes or needs for the time after rehabilitation. By classifying the results on the basis of a comparison group, conclusions are drawn for further projects on suitable aftercare strategies in the novel indication COVID-19. Further study could aim at maintaining long-term rehabilitation effects and maintaining or restoring employability. In addition, it may be possible that different recommendations are made for the varying severity of the disease course; therefore, further consideration is necessary.

\section{Immediate expected results of the project}

As this is an observational study, the baseline situation and the changes in the mentioned outcomes are described. These results are reported in relation to the comparison group, and statements on the extent to which there are similarities and differences are provided. The rehabilitation contents, needs and ability; aftercare recommendations and their implementation are also described descriptively for different degrees of disease severity.

Although the rehabilitants do not receive an intervention in the strict sense, they all undergo rehabilitation. Based on previous studies on pneumological rehabilitation as well as from the current experience of rehabilitation physicians, we expect positive changes in outcomes during rehabilitation. For example, we expect a significant reduction in the burden of disease and psychological impairments at the end of rehabilitation. The extent to which the effects on outcomes change over all four measurement points will be described in this study.

The results of the study will show whether rehabilitants have different needs in terms of rehabilitation, its contents and aftercare due to their individual course of illness, their previous illnesses or other personal prerequisites. With regard to aftercare, it is assumed that individual needs range from their own initiative and classic aftercare programmes to more intensive aftercare.

\section{Transferability of the project results to the everyday care situation}

In recent months, it has become apparent that the need for rehabilitation services for COVID-19 survivors is increasing. To offer a treatment that meets the needs of rehabilitants, appropriate research is necessary. At the end of the present study, practical recommendations for rehabilitation after COVID-19 will be developed. A common consensus in the rehabilitation procedure after COVID-19 can guarantee a universal standard in German rehabilitation.

The observational study is conducted in five German rehabilitation clinics. However, it is possible to transfer the results and provide recommendations to other pneumological rehabilitation clinics.

\section{Limitations}

To ensure equal treatment, all characteristics of the participants (e.g., age, education) and the disease (e.g., severity of the disease, disease status, and comorbidity) are documented and compared between groups. If differences arise between the two groups, they are comparably integrated in the analysis (e.g., as covariates in multivariate methods or by propensity score matching). Based on our present knowledge, we assume that structural changes in the context of this project are very unlikely.

\section{Abbreviations}

CG: Comparison group; COPD: Chronic obstructive pulmonary disease; COVID-19: Coronavirus disease; DRV Bund: Deutsche Rentenversicherung Bund; DSM-IV: Diagnostic and Statistical Manual of Mental Disorders; ICF: International Classification of Functioning, Disability and Health; 
ID: Identification number; IPD: Individual participant data; PHQ-D: Patient Health Questionnaire; PSM: Propensity score matching

\section{Acknowledgements}

Not applicable.

\section{Authors' contributions}

MR led the writing of this manuscript. JF, HBS, LD, TG, POS, AK and RD contributed to the overall study rationale, design and method development. In addition, RD conducted parts of the background literature review, assembled measures and critically reviewed the manuscript. All authors read and approved the manuscript. No professional writers were employed.

\section{Funding}

The funding for this project is provided by the German Pension Insurance, project number 0421/40-64-50-74. The funding body has no role in the study design, nor is it involved in the collection, analysis and interpretation of data or the writing of the manuscript. Significant protocol modifications, including changes to the work schedule, financial plan, project leader and planned investments, require approval by the funding body. Our application has undergone a review by an independent panel of experts. Open Access funding enabled and organized by Projekt DEAL.

\section{Availability of data and materials}

The datasets generated and/or analysed during the current study are not publicly available due to the data protection regulations and declarations of consent of the study participants. The declarations of consent exclude the release of the data for external analyses. A sample declaration of consent is available from the corresponding author on request. The study results are published and thus made available to all those interested in the study. The study protocol, the dataset at the participant level and the statistical code for generating the results are not publicly available.

\section{Declarations}

\section{Ethics approval and consent to participate}

This study will be performed in accordance with the Declaration of Helsinki. The ethical committee of the University of Lübeck approved the study for all cooperating rehabilitation-clinics (register number: 20-391) on 5 November 2020. The participants sign a consent form. The data protection concept is implemented according to the current guidelines of the German Pension Insurance ("Deutsche Rentenversicherung Bund", DRV Bund).

Before the study begins, all potential participants are informed in writing about the study and asked for their signed consent. Therefore, the documents (patient information sheet, declaration of consent) were prepared in accordance with the guidelines of the DRV Bund data protection officer $[43,44]$. Based on the General Data Protection Regulation (European Union), the participants may contact the competent authority for data protection and the contact person of the respective supervisory authority. With regard to data protection, we refer the study participants to the DRV Bund, which is in charge of this aspect of the study.

All personal data are pseudonymised in the rehabilitation clinics and stay password-protected in each clinic. Participants who meet the inclusion criteria and provide consent are included in the study. All personal data (questionnaire data) are pseudonymised by the clinic (using ID numbers). The assignment list (ID number and address) is kept and stored strictly separate from the questionnaire file. Immediately after completion of the twelvemonth follow-up and data control, this key file will be deleted, and the data are anonymised. The original documents will be kept in accordance with the Guidelines and Recommendations for Securing Good Epidemiological Practice (GEP) [45] for up to ten years after the end of the study.

\section{Consent for publication}

Not applicable because the manuscript does not contain any personal data.

\section{Competing interests}

The authors declare that they have no competing interests.

\section{Author details}

'Institute for Social Medicine and Epidemiology, Department Rehabilitation Sciences, University of Lübeck, Ratzeburger Allee 160, 23562 Lübeck,
Germany. ${ }^{2}$ MEDIAN Klinik Heiligendamm, Kinderstrand 1, 18209 Bad Doberan, Germany. ${ }^{3}$ Ostseeklinik Schönberg-Holm, An den Salzwiesen 1, 24217 Schönberg-Holm, Germany. ${ }^{4}$ Median Klinik Flachsheide Bad Salzuflen, Forsthausweg 1, 32105 Bad Salzuflen, Germany. ${ }^{5}$ Reha-Zentrum Schömberg, Römerweg 50, 75328 Schömberg, Germany. ${ }^{6}$ MEDIAN Klinik Flechtingen, Parkstraße, 39345 Flechtingen, Germany.

Received: 27 March 2021 Accepted: 12 April 2021

Published online: 22 April 2021

\section{References}

1. Robert Koch Institut. SARS-CoV-2 Steckbrief zur Coronavirus-Krankheit-2019 (COVID-19) 2020 [01.02.2021]. Available from: https://www.rki.de/DE/ Content/InfAZ/N/Neuartiges_Coronavirus/Steckbrief.html\#doc13776792 bodyText2.

2. Robert Koch Institut. Hinweise zu Erkennung, Diagnostik und Therapie von Patienten mit COVID-19. 2021 01.02.2021.

3. Schilling J, Lehfeld A-S, Schumacher D, Diercke M, Buda S, Haas W, et al. Krankheitsschwere der ersten COVID-19-Welle in Deutschland basierend auf den Meldungen gemäß Infektionsschutzgesetz. Journal of Health Monitoring. 2020(S11):1-20.

4. Grabowski DC, Joynt Maddox KE. Postacute Care Preparedness for COVID19: Thinking Ahead. JAMA. 2020.

5. Ngai JC, Ko FW, Ng SS, To K-W, Tong M, Hui DS. The long-term impact of severe acute respiratory syndrome on pulmonary function, exercise capacity and health status. Respirology. 2010;15(3):543-50.

6. Hui DS, Wong KT, Antonio GE, Tong M, Chan DP, Sung JJ. Long-term sequelae of SARS: physical, neuropsychiatric, and quality-of-life assessment. Hong Kong Med J. 2009;15 Suppl 8:21 - 3.

7. Mak IW, Chu CM, Pan PC, Yiu MG, Chan VL. Long-term psychiatric morbidities among SARS survivors. Gen Hosp Psychiatry. 2009;31(4):318-26.

8. Carfi A, Bernabei R, Landi F. Group ftGAC-P-ACS. Persistent Symptoms in Patients After Acute COVID-19. JAMA. 2020.

9. King's College London. ZOE Global Limited. How long does COVID-19 last?. 2020.

10. Thomas P, Baldwin C, Bissett B, Boden I, Gosselink R, Granger CL, et al. Physiotherapy management for COVID-19 in the acute hospital setting: clinical practice recommendations. Journal of Physiotherapy. 2020.

11. Reißhauer A, Baack A, Liebl ME. Physiotherapie bei erwachsenen Patienten mit Verdacht oder Nachweis von COVID-19 an der Charité Universitätsmedizin Berlin. Physikalische Medizin. 2020;30(02):64-5.

12. Li J. Rehabilitation management of patients with COVID-19: lessons learned from the first experience in China. Eur J Phys Rehabil Med. 2020;56(3):335-8.

13. Brugliera L, Spina A, Castellazzi P, Cimino P, Tettamanti A, Houdayer E, et al. Rehabilitation of COVID-19 patients. J Rehabil Med. 2020;52(4):jrm00046.

14. Gloeckl R, Buhr-Schinner H, Koczulla A, Schipmann R, Schultz K, Spielmanns $M$, et al. DGP-Empfehlungen zur pneumologischen Rehabilitation bei COVID-19. Pneumologie. 2020.

15. Khan F, Amatya B. Medical Rehabilitation in Pandemics: Towards a New Perspective. J Rehabil Med. 2020;52(4):jrm00043.

16. bij de Vaate E, Gerrits KHL, Goossens PH. Personalized recovery of severe COVID19: Rehabilitation from the perspective of patient needs. Eur J Clin Invest. 2020;50(7):e13325.

17. Deck R, Muche-Borowski C, Mittag O, Hüppe A, Raspe H. IMET - Index zur Messung von Einschränkungen der Teilhabe. In: Bengel J, Wirtz M, Zwingmann C, editors. Diagnostische Verfahren in der Rehabilitation. Göttingen: Hogrefe; 2008. pp. 372-4.

18. Deck R. Veränderungen von Teilhabestörungen nach Reha. Klinische Verhaltensmedizin Rehabilitation. 2007;78:229-36.

19. Deck R, Mittag O, Hüppe A, Muche-Borowski C, Raspe H. Index zur Messung von Einschränkungen der Teilhabe (IMET) - Erste Ergebnisse eines ICForientierten Assessmentinstrumentes. Klinische Verhaltensmedizin Rehabilitation. 2007;76:113-20.

20. Kazis LE, Miller DR, Skinner KM, Lee A, Ren XS, Clark JA, et al. Patientreported measures of health: The Veterans Health Study. J Ambul Care Manag. 2004;27(1):70-83.

21. Buchholz I, Feng Y-S, Buchholz M, Kazis L, Kohlmann T. The German Version of the Veterans Rand - 36/12 Item Health Survey: Translation and Adaptation of a Generic Health Assessment Instrument in a Sample of Inpatient Rehabilitation Patients2020. 
22. Nilges P, Essau C. Die Depressions-Angst-Stress-Skalen. Der Schmerz. 2015 29(6):649-57.

23. Spitzer RL, Kroenke K, Williams JB, Löwe B. A brief measure for assessing generalized anxiety disorder: the GAD-7. Arch Intern Med. 2006;166:1092-7.

24. Löwe B, Spitzer RL, Zipfel S, Herzog W. Gesundheitsfragebogen für Patienten (PHQ-D). Komplettversion und Kurzform. Testmappe mit Manual, Fragebögen, Schablonen. 2. Auflage. Karlsruhe 2002.

25. Gilbody S, Richards D, Brealey S, Hewitt C. Screening for depression in medical settings with the Patient Health Questionnaire (PHQ): a diagnostic meta-analysis. J Gen Intern Med. 2007;22(11):1596-602.

26. GlaxoSmithKline. COPD Assessment Test (CAT). 2009.

27. Jones PW, Harding G, Berry P, Wiklund I, Chen W-H. Kline Leidy N. Development and first validation of the COPD Assessment Test. Eur Respir J. 2009;34(3):648-54.

28. IImarinen J. The Work Ability Index (WAl). Occup Med. 2007:57(2):160.

29. Mittag O, Glaser-Möller N, Ekkernkamp M, Matthis C, Héon-Klin V, Raspe A, et al. Prädiktive Validität einer kurzen Skala zur subjektiven Prognose der Erwerbstätigkeit (SPE-Skala) in einer Kohorte von LVA-Versicherten mit schweren Rückenschmerzen oder funktionellen Beschwerden der inneren Medizin. Soz-Präventivmed. 2003;48:361-369.

30. Deck R, Raspe H. Regionale Qualitätssicherung in der medizinischen Rehabilitation. Qualitätsgemeinschaft medizinische Rehabilitation in SchleswigHolstein - Initiative und Erprobung. Rehabilitation. 2006;45:146-51.

31. Weis J, Tomaszewski KA, Hammerlid E, ignacio Arraras J, Conroy T, Lanceley A, et al. International psychometric validation of an EORTC quality of life module measuring cancer related fatique (EORTC QLQ-FA12). JNCI: Journal of the National Cancer Institute. 2017;109(5).

32. Kecke S, Ernst J, Einenkel J, Singer S, Hinz A. Psychometric Properties of the Fatigue Questionnaire EORTC QLQ-FA12 in a Sample of Female Cancer Patients. Journal of Pain symptom Management. 2017;54(6):922-8.

33. Deck R, Raspe H. Regionale Qualitätssicherung in der medizinischen Rehabilitation. Qualitätsgemeinschaft medizinische Rehabilitation in Schleswig-Holstein - Hauptstudie und Ergebnisse aus orthopädischen Kliniken. Rehabilitation 2006;45:272-81.

34. Mensink G. Körperliche Aktivität. Gesundheitswesen. 1999;61:126-31

35. Sangha O, Stucki G, Liang MH. The self administered comorbidity questionnaire: a new method to assess comorbidity for clinical and health services research. Arthr Rhuem. 2003;49:156-63.

36. Deck R, Röckelein E. Zur Erhebung soziodemografischer Indikatoren in den rehabilitationswissenschaftlichen Forschungsverbünden. In: Verband Deutscher Rentenversicherungsträger, editor. Förderschwerpunkt Rehabilitationswissenschaften: Empfehlungen der Arbeitsgruppen Generische Methoden, Routinedaten und Reha-Ökonomie. Frankfurt am Main: Verband Deutscher Rentenversicherungsträger; 1999. pp. 84-96.

37. Güell R, Casan P, Belda J, Sangenis M, Morante F, Guyatt GH, et al. Longterm Effects of Outpatient Rehabilitation of COPD: A Randomized Trial. CHEST. 2000;117(4):976-83.

38. Cote CG, Celli BR. Pulmonary rehabilitation and the BODE index in COPD. Eur Respir J. 2005;26(4):630-6.

39. Mayring P. Qualitative Inhaltsanalyse: Grundlagen und Techniken. Weinheim: Beltz-Verlag; 2010

40. Schreier M. Qualitative content analysis in practice. Los Angeles: Sage; 2012.

41. Schreier M. Varianten qualitativer Inhaltsanalyse: Ein Wegweiser im Dickicht der Begrifflichkeiten. Forum Qualitative Sozialforschung. 2014;15(1).

42. Kuckartz U. Qualitative Inhaltsanalyse: Methoden, Praxis, Computerunterstützung. Weinheim: Beltz Juventa; 2016.

43. DRV Bund. a [Available from: https://www.deutsche-rentenversiche-rung de/Allgemein/de/Inhalt/3_Infos_fuer_Experten/01_sozialmedizin_forschung/ downloads/reha_wissenschaften/Datenschutz_Hinweise.pdf?_blob=publica tionFile\&v=5.

44. DRV Bund. b [Available from: https://www.deutsche-rentenversiche-rung de/Allgemein/de/Inhalt/3_Infos_fuer_Experten/01_sozialmedizin_forschung/ downloads/reha_wissenschaf-ten/Datenschutz_Textbausteine_ Einwilligungserklaerung_3070.pdf?_blob=publicationFile\&v=8.

45. Hoffmann W, Latza U, Terschüren C. Leitlinien und Empfehlungen zur Sicherung von Guter Epidemiologischer Praxis (GEP) - überarbeitete Fassung nach Evaluation. Das Gesundheitswesen. 2005;67:217-25.

\section{Publisher's Note}

Springer Nature remains neutral with regard to jurisdictional claims in published maps and institutional affiliations.

Ready to submit your research? Choose BMC and benefit from:

- fast, convenient online submission

- thorough peer review by experienced researchers in your field

- rapid publication on acceptance

- support for research data, including large and complex data types

- gold Open Access which fosters wider collaboration and increased citations

- maximum visibility for your research: over $100 \mathrm{M}$ website views per year

At BMC, research is always in progress.

Learn more biomedcentral.com/submissions 